\title{
Evaluation of TSP for Emergency Routing
}

\author{
A. G. M. Zaman \\ Department of Computer Science, American International University-Bangladesh \\ E-mail: agmzaman@aiub.edu
}

\section{Sajib Hasan}

Department of Computer Science, American International University-Bangladesh

E-mail: sajib.hasan@aiub.edu

\author{
Mohammad Samawat Ullah \\ Department of Computer Science, American International University-Bangladesh \\ E-mail: samawat@aiub.edu
}

Received: 25 June 2020; Accepted: 07 September 2020; Published: 08 February 2021

\begin{abstract}
The paper considers the symmetric traveling salesman problem and applies it to sixty-four (64) districts of Bangladesh (with geographic coordinates) as a new instance of the problem of finding an optimized route in need of emergency. It approached three different algorithms namely Integer Linear Programming, Nearest-neighbor, and Metric TSP as exact, heuristic, or approximate methods of solving the NP-hard class of problem to model the emergency route planning. These algorithms have been implanted using computer codes, used IBM ILOG CPLEX parallel optimization, visualized using Geographic Information System tools. The performance of these algorithms also has been evaluated in terms of computational complexity, their run-time, and resulted tour distance using exact, approximate, and heuristic methods to find the best fit of route optimization in emergence thus contributing to the field of combinatorial optimization.
\end{abstract}

Index Terms: Integer Linear Programming, Nearest Neighbor, Metric TSP, Emergency Response Computational Tool and Models (ERT), Geographical Information System (GIS).

\section{Introduction}

Be it pandemic or disasters, it is predicted to continue and having a great impact on the socio-economic welfare of the entire world [1]. Authorities of emergency management service use several Emergency Response Computational Tool and Models (ERT) to route resources; however, advantages and limitations of such tools is not always clear [2]. Decision-support systems should consider demands, resources, priorities, supply chain, and infrastructure to take realtime decisions towards actions in mitigation, preparedness, response, and recovery phases of emergency management activities $[3,4]$.

The study address the problem that decision makers face in tour optimization in large-scale (all cities of the entire country) where the goal is to maximize social welfare in minimum time. While much of the TSP or resource routing analysis works on a predefined map, implemented within the framework of the spatial information provided, the study focuses on automating the construction process of a distance matrix from any given map.

The study illuminates computational aspects of finding the most efficient way to visit each one of many demand nodes once and returning to the single distribution node in emergency responses where resource routing becomes difficult. A well-known combinatorial optimization problem known as Travelling Salesman Problem has been used to find an optimized tour in such an emergency. In the classic TSP, the salesman must visit a fixed number of cities before returning to his original starting point. The objective is to choose the order of visit to minimize the travel distance $[5,6]$. The classical TSP setup has been applied to sixty-four (64) districts of Bangladesh to find an optimized tour where the objective of finding the minimum traveling distance with limited resources in an emergency becomes a lifesaving matter.

The main objective of the study is set to use several formulations of TSP as a computational tool to evaluate their performance and applicability to resources route optimization in emergency by minimizing total travelling distance with emerged constraints in emergence.

To clarify the scope of this study, the following constraints are put forward: (1) there is a shortage of resource during the emergency response; (2) all demands nodes are connected; (3) the resource is delivered by vehicle; (3) all demand nodes have same priority; (4) demand nodes can be served only once during a tour; and (5) the vehicle starts at 
the distribution center and returns to the same distribution center. Routing problems in emergency response determine which vehicle will visit which demand point in which sequence with the objective of minimizing the total distance traveled [7].

Throughout the study the authors hope to build a framework and propose the tool of finding the optimal tour in emergence utilizing exact, approximate, or heuristic methods of combinatorial optimization problem. The study also hope to achieve a visualization technique to recommend the resulted tour.

The rest of the paper is organized as follows. In Section II, we justify the Travelling Salesman Problem for resource routing problems in an emergency. In Section III, we describe three different TSP models in terms of their configuration and computational complexity. Computational results are reported in Section IV. Finally, some concluding remarks directions are presented in Section V.

\section{Related Works}

Strategic preparedness and response plan needed during pandemic (ex. COVID-19) or in any natural disaster $[2,3,4,15,16]$. The study raise the importance of resource routing for emergency response and apply TSP as a tool of emergency management system using geospatial data to plan optimal route thus reducing time and cost of resource distribution.

Resource routing during an emergency would be needed for delivering social or medical services to individuals at their homes rather than individuals coming to the service point [8]. Different organizations get involved, different kind of resources (consumable or non-consumable) may have to be provided, coordinated, and becomes a difficult activity during an emergency [9]. Thus, resource planning and routing becomes crucial to increase responsiveness.

Routing problems in an emergency may have to consider random events such as unavailability or variability of resources, uncertain environment, damaged transportation network, complex traffic system, available vehicles and their capacity, and dynamic demand change [9,10,11]. During these emergency situations Travelling Salesman Problem may be applied in small instances of resource routing with heterogeneous vehicles and demand points $[10,11,12]$. Cluster analysis can also be combined with Travelling Salesman Problem during this kind of emergency, where TSP is applied in each cluster of categorized customers with most similarities $[13,14]$. Disconnected disaster affected regions can be combined with single distribution center and TSP can be applied to each of these disconnected regions locally; satisfied by more than one vehicle in-terms of Vehicle Route Planning (VRP) $[15,16]$.

In an emergency, appropriate routing technology with the use of traditional routing algorithms traveling salesman problem can save lives [17]. With the main objective of finding the optimized tour in any scale of instances, it is important to consider additional objective functions and constraints.

\section{TSP Formulation and Computational Complexity}

Finding the shortest route in large solution space (in our instance it is 64 districts of Bangladesh) using the classical TSP becomes NP-hard of combinatorial problem [23,24,25]. Among many formulations of TSP, the study use Integer Linear Programming (ILP) for exact optimal solution and Nearest Neighbor Algorithm (NNA) for near optimal solution to identify the dominance among them during emergency response by comparing results $[19,25,33,40]$.

Given Sixty-Four (64) demand points to finding the most efficient way to visit them for distributing resources becomes an optimization problem. Solving the optimization problem with a large solution space is NP-hard or NPcomplete when we calculate every possible solution to choose the best solution [6]. For our instance, finding the shortest tour of all sixty-four districts of Bangladesh using the classic traveling salesman problem thus requires exponential time [6,7].

There exist many formulations of the TSP even in symmetric and asymmetric form [18,19]. This paper analyzes and compares two polynomial and one exponential formulation of the traveling salesman problem. The exact optimal solution has been found using Integer Linear Programming formulation then compared with two other approximate solution procedures namely the Nearest Neighbor Algorithm and Metric formulation of the TSP. These three different formulations and their computational complexity have been discussed in this section.

\subsection{Representation of TSP}

There are $n$ demands nodes as a set $A=\{1,2, \ldots, n\}$, distance between any two nodes is $C_{i j}=n$ (where, $0<n \leq$ 757) and distance among all cities are stored in $n \times n$ distance matrix; whether they are connected directly or not. The following notation is used:

$n=$ number of demand nodes to be visited

$\mathrm{i}, \mathrm{j}=$ indices of nodes between 1 to $\mathrm{n}$ integer

$\mathrm{x}_{\mathrm{ijt}}= \begin{cases}1 & \text { if exists edge between } \mathrm{i} \text { and } \mathrm{j} \text { in step } \mathrm{u}_{\mathrm{i}} \\ 0 & \text { otherwise }\end{cases}$

$\mathrm{c}_{\mathrm{ij}}=$ cost or distance between node $\mathrm{i}$ to $\mathrm{j}$ 


\section{$\mathrm{u}_{\mathrm{i}}=$ steps to visit city $\mathrm{i}$}

The optimal solution is the lowest total distance traveled in the hypothetical tour. The objective function $(\mathrm{O})$ is a minimization of the total sum of costs (distances) of all the chosen elements of the tour:

$$
\text { Minimize } o=\sum_{i \in A} \sum_{\substack{j \neq i \\ j \in A}} c_{i j} x_{i j}
$$

For all step of $u_{i}$, there exists only one edge and more than one pair of nodes is not possible to visit in each step, the tour is subject to the following constraint-

$$
\sum_{\substack{i \in A \\ i \neq j}} x_{i j}=1, \quad \text { given } j \in A
$$

For each node $i$, there is only one other node $j$ which is being reached, at some time, hence the constraint:

$$
\sum_{\substack{j \in A \\ j \neq i}} x_{i j}=1, \quad \text { given } i \in A
$$

The node, which is reached at step $u_{i}$, will not be included in step $u_{i}+1$ of the tour to exclude disconnected subtours. This constraint of sub-tour elimination can be formulated as:

$$
\left(u_{i}-u_{j}\right)+(n-1) x_{i j} \leq(n-2) \forall_{i, j \in A}, i \neq j
$$

\subsection{ILP Formulation}

The exact solution approach of TSP would evaluate all possible combinations of $\mathrm{n}$ demand nodes. For $\mathrm{n}$ nodes, there are (n-1)! possible number of permutations thus exists (n-1)! Routes [20,21]. The sub-tour-elimination constraint raises a problem where for $n$ demand nodes, the number of disjoints sub tour constraint gets added [22,23]. This make the TSP formulation large integer-programming problem [23,24,25].

When TSP becomes a linear program with a linear objective function (minimization of distance or cost) having linear inequality constraints; there are Simplex method and Interior Point method available among many popular methods for solving TSP with Integer Linear Programming formulation [25,26].

To apply linear programming solution procedure for TSP this paper applies the Simplex algorithm for relaxations of the problem to find optimal solutions, which seems to be a good choice according to some literature review $[26,27,28]$. The study even steps further to use IBM's CPLEX optimizer which enables decision optimization for improving efficiency $[29,30]$.

Once determined that using the Simplex algorithm for the linear programming solution procedure of TSP would give an optimal solution, there is the branch and bound method to determine the integer optimality $[27,28]$. Branch and Bound method is a search procedure were for the upper bound it uses the non-integer solution and search for possible integer solutions to determine the lower bound. Optimality is achieved hen the upper and lower bound coincide [31,32].

During this study, using the above-mentioned mathematical formulation an integer programming procedure was used to result in the optimal solution with the IBM's CPLEX optimizer.

\subsection{NNA Formulation}

Exponential nature of Integer Linear Programming approach of TSP can be dealt with the approximate approach of finding near-optimal solution rather than optimal solution [33]

The Nearest Neighbor heuristic is simple and consider the "greedy" approach thus returns solution in reasonable computing time (polynomial time) but does not always provide even near-optimal solution [33,34]. Emergency routing would require a faster response but it also demands good solution, thus the paper evaluates the Nearest Neighbor Heuristic by two important criteria; they are: (1) the computational time required to reach the solution; and (2) performance in differences of distance concerning the optimal solution.

The algorithm simply selects the least-cost edge among the unvisited neighbor nodes to construct a tour repeatedly until all nodes are visited $[35,36]$. The complete path is returned as a solution once there is no more unvisited node. The path is generated as follows: 
1. Find the nearest unvisited node $X$ from any Node

2. Flag $\mathrm{X}$ as visited.

3. Terminate, if remain no unvisited node.

4. Else set node B as the current node.

5. Go to step 1 .

\subsection{Metric TSP}

The study looks further to improve the efficiency or optimality of finding the solution and take another approach namely Metric TSP. After further literature review $\alpha$-approximation algorithm seems to be feasible, whose objective value would be always a factor of $\alpha$ from the best outcome [20,38]. Applying triangular inequality as a restriction, the problem becomes metric TSP, for which a constant factor (metric) of approximation would exist [38].

The tree doubling algorithm seems to be a good choice of 2-approximation algorithm for Metric TSP, which computes a Hamiltonian tour by using a minimum spanning tree [38,39]. A minimum spanning tree can efficiently be computed via Prim's or Kruskal's Algorithm [38].

Using a Lemma and Theorem it can be proved that the running time of Metric TSP is dominated by running time required to compute a minimum spanning tree $[38,40]$. This is polynomial since the minimum spanning tree is polynomial [38]. The study implements the Metric TSP to verify the solution.

\section{Experimental Setup and Methodology}

The experimental process expect to establish a model of automating the process of constructing distance matrix of TSP problem and visualizing resulted optimal tour as recommendation for emergency management through implementation of ILP, NNA, and Metric TSP formulation. There is Data Acquisition \& Preparation step to produce one-dimensional array of nodes and distance matrix, Problem formulation step to implement Java representation of three different TSP formulation, Result comparison step to compare resulted tour, and Visualization technique as recommendation of optimal tour.

To identify the applicability of our proposed model in emergency routing a step by step procedure was followed to build the model itself. The model has been implemented programmatically using Java programming language to ensure its applicability. The output results of the programming model has then been visualized as a recommendation of the route in an emergency. Three different TSP formulation of section three of this study has been applied to the dataset to compare and analyze results. This section describes the whole process and a flow diagram is given below -

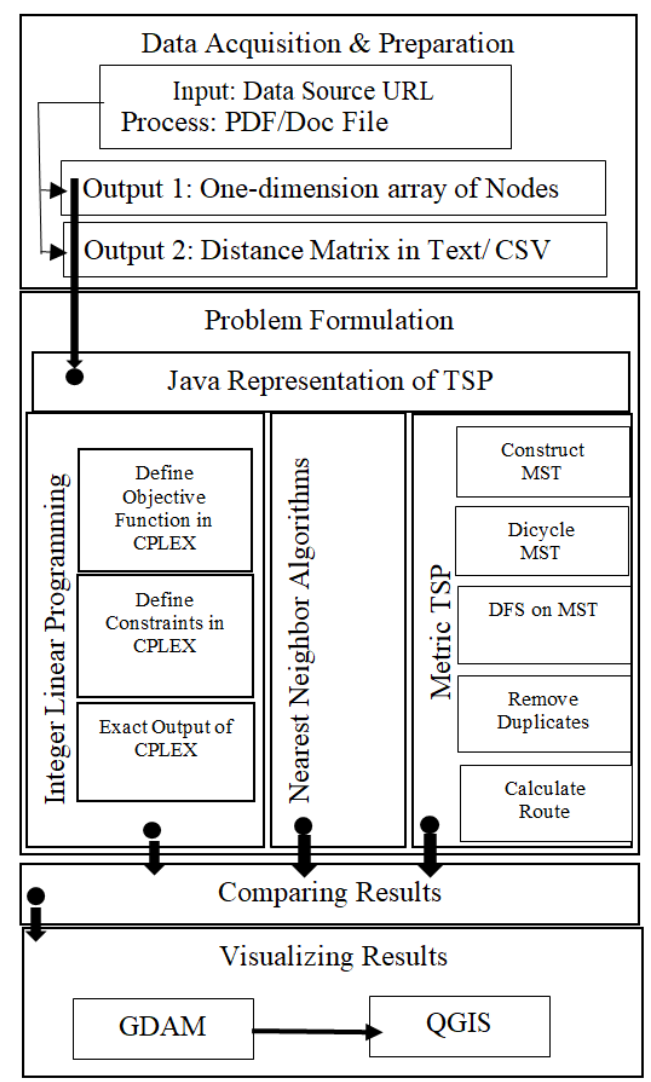

Fig.1. Proposed Model 


\subsection{Data Acquisition \& Preparation}

Most of the study about TSP or resource routing works on a predefined map, implemented in the context of given spatial information. The study focusses on automating the process of constructing the distance matrix from any given source.

A dataset of distances among sixty-four (64) cities of Bangladesh was generated on which the exact solution and heuristic approaches were tested. There were no available text data of the distance matrix of cities of Bangladesh but only in pdf format. From Road Transport and Highways Division of Bangladesh, a pdf file was captured and converted to a text file. A small Java program was created to automate the process. Results and the process is discussed in Section $\mathrm{V}$ of the study.

\subsection{Solution Procedure}

First, an exact enumeration procedure was implemented in a Java program using IBM's CPLEX Optimizer for efficient computation. Objective function and the three constraints for the Integer Linear Programming approach have been coded in CPLEX Model and integrated in Java program.

The computation in CPLEX Model for the exact solution took a while thus the other two approximation models proceeded for implementation with two other Java procedures. The Nearest Neighbor Algorithms were implemented using the pseudocode discussed in section 3.3 of the study. Once the NNA solution has been found where the tour difference from the optimal one was quite high, the Metric TSP algorithm was applied to find a better solution.

The solution procedure for 2-approximation Metric TSP was quite lengthy but gave better results than NNA implementation in polynomial time. The study produced a Minimum Spanning Tree from the graph of the distance matrix using the Kruskal Algorithm of MST [41,42]. Once found MST the study checked for any existing cycle [41]. A depth-first search was applied to the Minimum Spanning Tree to produce the DFS tree. Then duplicate nodes were removed from the resulted DFS tree to calculate route. A preorder traversal of the DFS resulted in the tour.

\subsection{Comparing Results}

The output of each algorithm has then been compared in terms of their run time and tour distance. Also, the sequence of nodes to visit has been produced as the output of each algorithm to map it to visualization.

\subsection{Visualizing Results}

The output of the optimal tour solution has been visualized with a directed edge on a colored country map of Bangladesh. To visualize the tour, geographic data (with latitude and longitude) of the country has been used, which is available in the Global Administrative Areas database [43]. Retrieved data from GDAM has then been used to plot the travel route on top of the geographic data and develop a tour on the map, using an opensource geographic data processing software named QGIS [44].

To draw arc from one node to another, latitude and longitude of the center of each node has been used. The resulted tour of the three different formulations (ILP, NNA, Metric TSP) of TSP has been placed on the foreground of the map of Bangladesh as a vector layer using three different shapefiles. A shapefile is a flat, nontopological system for storing geometric location and attributing geographic character information [45].

\section{Results and Discussions}

Academic solvers expect perfection and therefore take a very long time to determine optimal solutions and that would not work in response to an emergency. To route resources in emergency finding a solution in a fraction of time is necessary and a near-optimal solution with route optimization algorithms would work.

A good heuristic must provide near-optimal solutions, be easy to implement, flexible, and ultimately provide solutions in a reasonably short time [33,34,39]. This section shows the outcome of the procedures that have been followed in section four of the study.

\subsection{Data Source to Distance Matrix}

The output of the process discussed in section 4.1 of this study initially was a text file where rows are indexed by sixty-four distinct district names of Bangladesh. Figure 2 below is a part of the file. 


\begin{tabular}{|l|c|c|c|c|c|c|c|c|}
\hline & Dhaka & Bagerhat & Bandarban & Barguna & Barisal & Bhola & Bogra & Brahmanbaria \\
\hline Dhaka & 0 & 178 & 316 & 247 & 169 & 205 & 197 & 109 \\
\hline Bagerhat & 178 & 0 & 437 & 160 & 119 & 155 & 328 & 283 \\
\hline Bandarban & 316 & 437 & 0 & 469 & 391 & 427 & 510 & 301 \\
\hline Barguna & 247 & 160 & 469 & 0 & 84 & 120 & 445 & 352 \\
\hline Barisal & 169 & 119 & 391 & 84 & 0 & 36 & 366 & 273 \\
\hline Bhola & 205 & 155 & 427 & 120 & 36 & 0 & 402 & 309 \\
\hline Bogra & 197 & 328 & 510 & 445 & 366 & 402 & 0 & 274 \\
\hline Brahmanbaria & 109 & 283 & 301 & 352 & 273 & 309 & 274 & 0 \\
\hline
\end{tabular}

Fig.2. Initial text file produced from the process discussed in section 4.1

The file had sixty-five (65) columns and rows were sorted in lexicographical order of the city names. Index names were stored into a one-dimensional string array and a 64 by 64 integer array was used to create distance matrix to store distances among cities. This was a square matrix where an identical index of rows and columns correspond to the name of cities stored in the one-dimensional string array created earlier. A programming code in Java has been developed to automate the process of creating this distance matrix from a pdf file of a web source.

\subsection{Comparison of Results}

Integer Linear Programming formulation of the study which has been discussed in sections 3.1 and 4.1, solved the problem of finding an optimal tour in 2510.61 seconds (41.84 minutes) with a minimum total distance of 4059.0 kilometers. The minimum distance returned from the Nearest Neighbor Algorithm was 5189 kilometers, and from Metric TSP the was 5096 kilometers. These results were produced using Core i3, $2.8 \mathrm{GHz} \mathrm{CPU}$ with Ram speed of 5 GB on the Windows 10 operating system. Table 1 below shows the comparisons of different algorithms.

Table 1. Result Comparison

\begin{tabular}{|c|c|c|c|}
\hline & ILP & NNA & Metric TSP \\
\hline Total Distance & 4059 & $5189 \mathrm{Km}$. & $5096 \mathrm{Km}$. \\
\hline Time complexity & Exponential $(43 \mathrm{~min})$ & Polynomial & Polynomial \\
\hline Approximation Ration & Optimum & - & 2 approximation \\
\hline Difference & & $1130 \mathrm{Km}$. & $1037 \mathrm{Km}$. \\
\hline
\end{tabular}

Also, tour matrix was created for every resulted output of the three different TSP formulation. Table 2 shows a part of the matrix resulted from ILP formulation of TSP.

Table 2. Part of tour matrix resulted from ILP

\begin{tabular}{|c|c|c|c|}
\hline Source & Destination & Source & Destination \\
\hline Dhaka & Gazipur & Chandpur & Lakshmipur \\
\hline Gazipur & Tangail & Lakshmipur & Noakhali \\
\hline Tangail & Sirajganj & Noakhali & Feni \\
\hline Sirajganj & Manikganj & Feni & Chittagong \\
\hline Manikganj & Rajbari & Chittagong & Rangamati \\
\hline Rajbari & Faridpur & Rangamati & Bandarban \\
\hline
\end{tabular}

\subsection{Visualization of Results}

When it is about emergency routing visualizing the resulted route would help anyone to take decision and follow the resulted path $[46,47]$. This study visualizes the resulted tour as guidance during an emergency. Figure 3 below shows the drawn arcs resulted as a directed tour of the ILP formulation of TSP using the process discussed in section 4.4 of the study. 


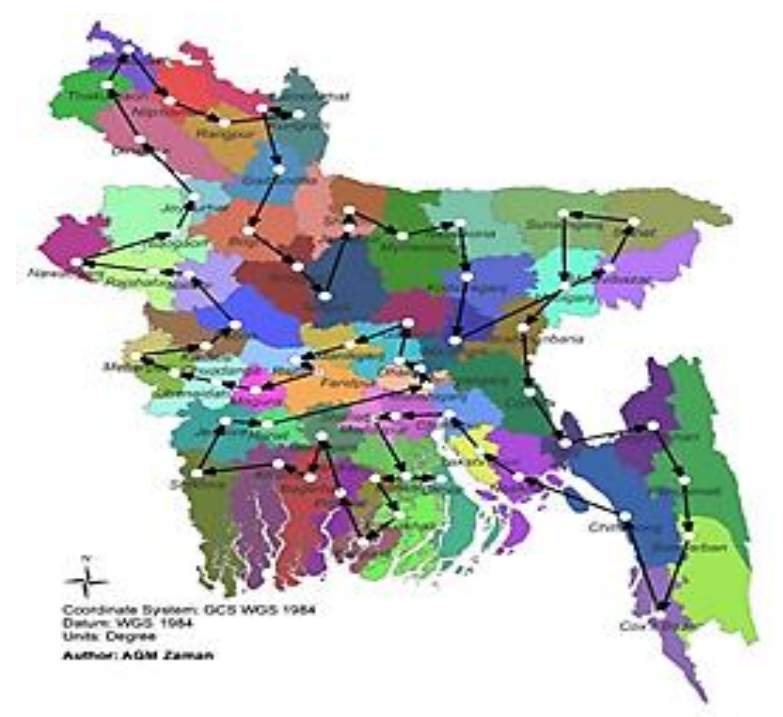

Fig.3. Directed tour resulted from ILP

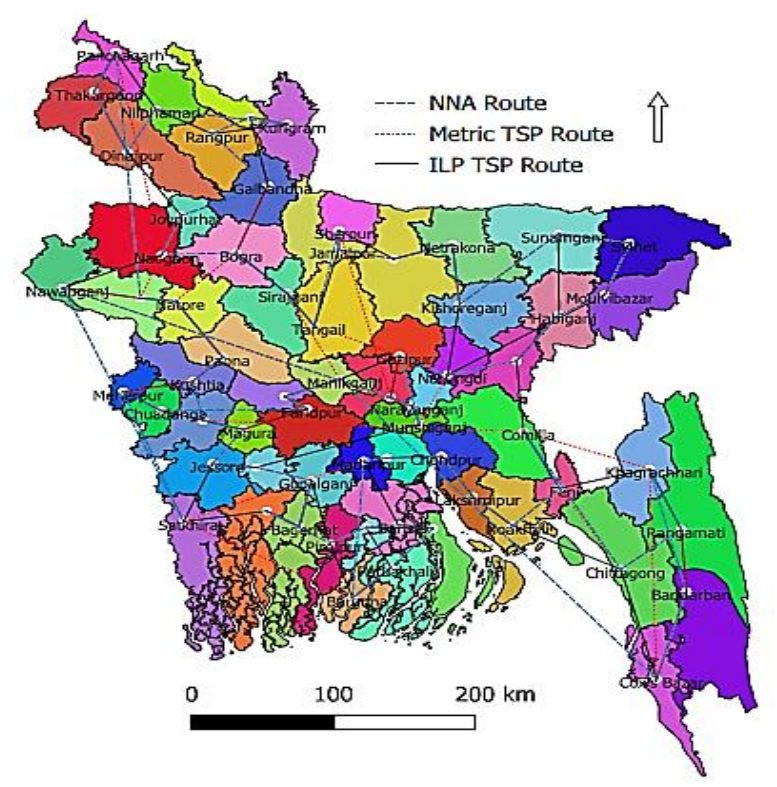

Fig.4. Visualizing Route of ILP, NNA, \& Metric TSP

Also, the route of the ILP, NNA, and Metric TSP has been plotted on the same map to visualize at the same time. Figure 4 shows the result.

Results discussed in Section 5.1 achieve one of the objectives of the study to automate the process of distance matrix construction from any given map. Comparison of results in section 5.2 of the study helps decision makers by returning optimal resources route in emergency through minimization of total travelling distance in minimum time thus achieve the other objective of the study. Visualization of all resulted route on the given map discussed in section 5.3 achieve another objective of recommending optimal tour to take decision in emergence.

\section{Conclusions}

The efficiency of logistic support during emergency heavily depends on route optimization. The strategic design of emergency routing for logistic network (road or railway) using a geographic coordinate system is effective when resources are limited. The Geographic Information System (GIS) feature (geographic coordinate) of the country of Bangladesh is a new instance of traveling salesman problems to find a sequence of demand nodes to be visited systematically. The paper used this new instance of TSP to derive optimal and near-optimal routing solutions to efficiently tackle emergency response operations on large areas.

An optimal solution to a difficult problem or NP-hard class of problems is unlikely to reveal in polynomial time. Therefore, optimization problems like traveling salesman problems are solved using exact, approximate, or heuristic methods. The paper approached all these three techniques to compare the results. To find the optimal result it used 
IBM's CPLEX. IBM ILOG CPLEX allowed solving the problem utilizing Integer Linear Programming algorithms with parallel optimization.

The efficiency of logistic approaches in emergency has been shown in early studies $[2,4,49]$. Several recent works modeled the emergency tour planning as TSP [9,50] or show connections with different TSP formulation [11,12,51]. Many academic researchers attempt to include GIS services in TSP and a future work of this study aims to do so to better exploit GIS features.

The study shows the usefulness of the travelling salesman problem in emergency routing using geographic information through quantitative assessment of the proposed framework. This is an advancement of application to the first Romanian TSP instance. Future work of the study should overcome the limitation of not being able to construct an automated decision support system for complete logistic planning in emergency.

\section{References}

[1] Annetta Burger,Talha Oz, William G. Kennedy, and Andrew T. Crooks, Computational Social Science of Disasters: Opportunities and Challenges, IJCAI,26 April 2019.

[2] Huibo Bi, Erol Gelenbe, Emergency Management Systems and Algorithms: A Comprehensive Survey, arXiv®, Cornell University, 21 Jun 2019.

[3] World Health Organization, 2019 Novel Coronavirus (2019-nCoV): STRATEGIC PREPAREDNESS AND RESPONSE PLAN, Creative Commons Attribution-NonCommercial-ShareAlike, 3 February 2020.

[4] National Research Council et al, Successful Response Starts with a Map: Improving Geospatial Support for Disaster Management, Chapter: 3 Emergency Management Framework, 2007, Page 47-80.

[5] Hoffman K.L., Padberg M., Rinaldi G. (2013) Traveling Salesman Problem. In: Gass S.I., Fu M.C. (eds) Encyclopedia of Operations Research and Management Science. Springer, Boston, MA.

[6] Thomas H. Cormen, Thomas H Cormen, Charles E Leiserson, Ronald L Rivest, Clifford Stein, Introduction to Algorithms, MIT Press, 2001, Page 1012-1013.

[7] Suzanne Ma, Understanding the Travelling Salesman Problem (TSP), Routific, January 2, 2020.

[8] Changshi Liu \& Gang Kou \& Yi Peng \& Fawaz E. Alsaadi, 2019. "Location-Routing Problem for Relief Distribution in the Early Post-Earthquake Stage from the Perspective of Fairness," Sustainability, MDPI, Open Access Journal, vol. 11(12), pages 1-16, June.

[9] Yi Hong, Deying Li, Qiang Wu, and Hua Xu1, Dynamic Route Network Planning Problem for Emergency Evacuation in Restricted-Space Scenarios, Journal of Advanced Transportation, 27 Jun 2018

[10] Viana Céspedes, V. (2018.). Optimization in the planning of forest harvesting services. Master's Thesis. University of the Republic (Uruguay). Faculty of Engineering.

[11] M. Assaf and M. Ndiaye, "Solving an Open Path Multiple Depot Multiple Traveling Salesman Problem after transformation," 2017 7th International Conference on Modeling, Simulation, and Applied Optimization (ICMSAO), Sharjah, 2017, pp. 1-4, doi: 10.1109/ICMSAO.2017.7934866.

[12] Igor Averbakh \& Wei Yu, 2020. "Multi-depot traveling salesmen location problems on networks with special structure," Annals of Operations Research, Springer, vol. 286(1), pages 635-648, March.

[13] Kusumahardhini, N., Hertono, G. F., \& Handari, B. D. (2020). Implementation of K-Means and crossover ant colony optimization algorithm on multiple traveling salesman problem. Journal of Physics: Conference Series, 1442(1), [012035]. https://doi.org/10.1088/1742-6596/1442/1/012035

[14] Anaya Fuentes GE, Herna'ndez Gress ES, Seck Tuoh Mora JC, Medina Marı'n J (2018) Solution to travelling salesman problem by clusters and a modified multi-restart iterated local search metaheuristic. PLoS ONE 13(8): e0201868. https:// doi.org/10.1371/journal.pone.0201868

[15] N. Gupta, T. Gupta, S. Samaddar and S. Roy, "WebReLog: A Web-based Tool for Disaster Relief Logistics with Vehicle Route Planning," 2019 IEEE International Conference on Systems, Man and Cybernetics (SMC), Bari, Italy, 2019, pp. 1012-1017, doi: 10.1109/SMC.2019.8913925.

[16] P. Ganguly and S. Roy, "Post-disaster relief by vehicle route planning and service time estimation in case of Chennai floods," 2017 4th International Conference on Information and Communication Technologies for Disaster Management (ICT-DM), Münster, 2017, pp. 1-8, doi: 10.1109/ICT-DM.2017.8275694.

[17] Gloria Cerasela Crisan, Camelia Pintea,and PinteaVasile Palade, Emergency management using geographic information systems: application to the first Romanian traveling salesman problem instance, April 2016, Knowledge and Information Systems 50(1):1-21, DOI: 10.1007/s10115-016-0938-8.

[18] K. Ilavarasi and K. S. Joseph, "Variants of travelling salesman problem: A survey," International Conference on Information Communication and Embedded Systems (ICICES2014), Chennai, 2014, pp. 1-7, doi: 10.1109/ICICES.2014.7033850.

[19] Padberg, M., Sung, T. An analytical comparison of different formulations of the travelling salesman problem. Mathematical Programming 52, 315-357 (1991). https://doi.org/10.1007/BF01582894

[20] Matthew Chatting, A Comparison of Exact and Heuristic Algorithms to Solve the Travelling Salesman Problem, The Plymouth Student Scientist, 2018, 11, (2), 53-91

[21] Michael Hahsler, Kurt Hornik, TSP Infrastructure for the Traveling Salesperson Problem, Journal of Statistical Software, December 2007, Volume 23, Issue 2.

[22] Kevin M. Curtin, Gabriela Voicu Matthew T. Rice, Anthony Stefanidis, A Comparative Analysis of Traveling Salesman Solutions from Geographic Information Systems, Wiley, 09 June 2013, https://doi.org/10.1111/tgis.12045

[23] Chapter 9, Integer Programming and Combinatorial Optimization, MIT Open Courseware, MIT Course Number 15.083J / $6.859 \mathrm{~J}$ 
[24] C. E. Miller, Albert W Tucker, R A Zemlin, Integer Programming Formulation of Traveling Salesman Problems, Journal of the ACM,October 1960, https://doi.org/10.1145/321043.321046

[25] Graver, J.E. On the foundations of linear and integer linear programming I. Mathematical Programming 9, $207-226$ (1975). https://doi.org/10.1007/BF01681344

[26] Andersen ED, Gondzio J, Mészáros C, Xu X (1996) Implementation of interior point methods for large scale linear programming. In: Terlaky $\mathrm{T}$ (ed) Interior Point Methods in Mathematical Programming.

[27] Rujira Visuthirattanamanee, Krung Sinapiromsaran,and Aua-aree Boonperm, Self-Regulating Artificial-Free Linear Programming Solver Using a Jump and Simplex Method, mathematics- MDPI, 5 March 2020

[28] J. Zhu, S. Qian and T. Shi, "Simplex Method of Computer Algorithms Practice," 2010 Second International Conference on Computer Research and Development, Kuala Lumpur, 2010, pp. 756-759, doi: 10.1109/ICCRD.2010.169.

[29] CPLEX Optimizer, IBM, https://www.ibm.com/analytics/cplex-optimizer

[30] N. Fares, M. Lebbar and N. Sbihi, "Quick response in fast fashion retail: An optimization supply chain responsiveness model," 2018 4th International Conference on Optimization and Applications (ICOA), Mohammedia, 2018, pp. 1-5, doi: 10.1109/ICOA.2018.8370565.

[31] E.M.L.Beale, Branch and Bound Methods for Mathematical Programming Systems, Annals of Discrete Mathematics, Volume 5, 1979, Pages 201-219

[32] David Applegate, Robert Bixby, Vasek Chvatal, William Cook, On the Solution of Traveling Salesman Problem, Documenta Mathematica-Extra Volume ICM 1998-III, 645-656

[33] Gözde Kizilateş, Fidan Nuriyeva, Chapter on the Nearest Neighbor Algorithms for the Traveling Salesman Problem, Advances in Computational Science, Engineering and Information Technology, 2013, Volume 225

[34] E. O. Asani, A. E. Okeyinka and A. A. Adebiyi, "A Construction Tour Technique For Solving The Travelling Salesman Problem Based On Convex Hull And Nearest Neighbour Heuristics," 2020 International Conference in Mathematics, Computer Engineering and Computer Science (ICMCECS), Ayobo, Ipaja, Lagos, Nigeria, 2020,pp.14,doi:10.1109/ICMCECS47690.2020.240847.

[35] Stefan Hougardy, Mirko Wilde, On the nearest neighbor rule for the metric traveling salesman problem, 27 March 2014 Discrete Applied Mathematics, Elsevier B.V.

[36] S. Dhakal and R. Chiong, "A hybrid nearest neighbour and progressive improvement approach for Travelling Salesman Problem," 2008 International Symposium on Information Technology, Kuala Lumpur, 2008, pp. 1-4, doi: 10.1109/ITSIM.2008.4631549.

[37] A H Ismail et al, Domino algorithm: a novel constructive heuristics for traveling salesman problem, 2019, IOP Conference Series: Materials Science and Engineering.

[38] Bläser M. (2008) Metric TSP. In: Kao MY. (eds) Encyclopedia of Algorithms. Springer, Boston, MA

[39] Chandra Chekuri, Kent Quanrud, Fast Approximations for Metric-TSP via Linear Programming, 5 Feb 2018, arXiv: 1802.01242 [cs.DS]

[40] Vladimir Deineko, Alexander Tiskin, Fast minimum-weight double-tree shortcutting for Metric TSP: Is the best one good enough?, 1 Oct 2007, arXiv:0710.0318 [cs.DS]

[41] Christopher Clapham and James Nicholson, The Concise Oxford Dictionary of Mathematics (4 ed.), 2013, Oxford University Press.

[42] Haiming Li, Qiyang Xia, Yong Wang, Research and Improvement of Kruskal Algorithm, October 2017, Journal of Computer and Communications, Vol.5 No.12.

[43] Global Administrative Areas (2012). GADM database of Global Administrative Areas, version 2.0. [online] URL: www.gadm.org

[44] QGIS.org (2020). QGIS Geographic Information System. Open Source Geospatial Foundation Project. http://qgis.org

[45] ESRI 2017. ArcGIS Desktop: Release 10. Redlands, CA: Environmental Systems Research Institute.

[46] Jennex, Murray E., Using Social and Information Technologies for Disaster and Crisis Management, Jan 31, 2013 , IGI Global.

[47] Sisi Zlatanova, Jonathan Li, Geospatial Information Technology for Emergency Response, Jan 24, 2008, CRC Press.

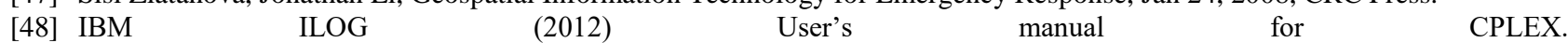
$\mathrm{ftp} / /$ public.dhe.ibm.com/software/websphere/ilog/docs/optimization/cplex/ps_usrmancplex

[49] Kemball-Cook D, Stephenson R (1984) Lessons in logistics from Somalia. Disasters 8:57-66

[50] Kirac E et al (2015) The traveling salesman problem with imperfect information with application in disaster relief tour planning IIE Trans 47(8):783-799

[51] Wex F et al (2014) Emergency response in natural disaster management: allocation and scheduling of rescue units. Eur J Oper Res 235(3):697-708

\section{Authors' Profiles}

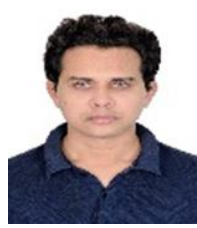

A. G. M. Zaman: Assistant Professor, Department of Computer Science in American International UniversityBangladesh (AIUB), interested in Data Mining, Machine Learning, and Algorithms. 
Sajib Hasan: Assistant Professor of school of computer science in American International University-Bangladesh (AIUB), interested in Algorithms, Artificial Intelligence, and Deep Learning.

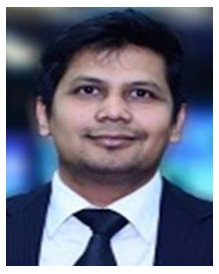

Mohammad Samawat Ullah: Assistant Professor of school of computer science in American International University-Bangladesh (AIUB), interested in Machine Learning, Deep Learning, Cyber Security.

How to cite this paper: A. G. M. Zaman, Sajib Hasan, Mohammad Samawat Ullah, "Evaluation of TSP for Emergency Routing", International Journal of Information Technology and Computer Science(IJITCS), Vol.13, No.1, pp.44-53, 2021. DOI: 10.5815/ijitcs.2021.01.03 